\title{
Efeitos da Analgesia do Sufentanil por Via Subaracnóidea e Bupivacaína a $0,25 \%$ por Via Peridural sobre as Concentrações Plasmáticas de Ocitocina e Cortisol em Gestantes em Trabalho de Parto *
}

\author{
Effects of Analgesic Intrathecal Sufentanil and 0.25\% Epidural \\ Bupivacaine on Oxytocin and Cortisol Plasma Concentration in \\ Labor Patients
}

Renato Mestriner Stocche, TSA ${ }^{1}$, Luiz Vicente Garcia, TSA ${ }^{2}$, Jyrson Guilherme Klamt, TSA ${ }^{2}$

\begin{abstract}
RESUMO
Stocche RM, Garcia LV, Klamt JG - Efeitos da Analgesia do Sufentanil por Via Subaracnóidea e Bupivacaína a 0,25\% por Via Peridural sobre as Concentrações Plasmáticas de Ocitocina e Cortisol em Gestantes em Trabalho de Parto
\end{abstract}

Justificativa e Objetivos - O sufentanil por via subaracnóidea promove analgesia do trabalho de parto comparável à peridural com anestésicos locais. As duas técnicas diminuem alguns parâmetros da resposta neuroendócrina à dor do parto, e ambas podem atenuar a liberação reflexa de ocitocina em animais. O objetivo deste estudo foi comparar os efeitos das duas técnicas nas concentrações plasmáticas de ocitocina (CPO) e cortisol (CPC) durante o trabalho de parto.

Método - Trinta parturientes com $4-7 \mathrm{~cm}$ de dilatação foram arroladas neste estudo de forma aleatória e encoberta. No grupo $S$ receberam sufentanil $(10 \mu \mathrm{g})$ por via subaracnóidea e no grupo $B$ receberam bupivacaína a $0,25 \%$ por via peridural. A analgesia foi medida usando Escala Analógica Visual e amostras sangüíneas de ocitocina e cortisol foram obtidas antes, 30 e 60 minutos após a administração das drogas. As CPO e CPC foram determinadas através da técnica de radioimunoensaio.

Resultados - $A$ analgesia no grupo $S$ foi mais intensa aos 30 minutos. As CPO e CPC nos dois grupos foram semelhantes antes da analgesia. No grupo $S$, houve diminuição significativa da $C P O$ aos 60 minutos e CPC aos 30 e 60 minutos, enquanto no grupo $B, C P O$ e CPC permaneceram estáveis durante todo $o$ estudo.

Conclusões - Nas condições deste estudo, a analgesia peridural com bupivacaína esteve associada à manutenção das concentrações plasmáticas de cortisol e ocitocina. Contrariamente, a analgesia promovida pelo sufentanil, por via subaracnóidea, foi mais intensa e produziu diminuição nas concentrações destes hormônios.

UNITERMOS - ANALGESIA: parto; ANALGÉSICOS, Opióides: sufentanil; HORMÔNIOS: cortisol, ocitocina; TÉCNICAS ANESTÉSICAS, Regional: peridural

\footnotetext{
* Recebido da (Received from) Maternidade Mater do Hospital de Clínicas da USP, Ribeirão Preto, SP

1. Médico Assistente do Hospital de Clínicas da USP

2. Professor da Faculdade de Medicina da USP, Ribeirão Preto, SP

Apresentado (Submitted) em 13 de novembro de 2000

Aceito (Accepted) para publicação em 13 de fevereiro de 2001

Correspondência para (Mail to)

Dr. Renato Mestriner Stocche

Rua Adolfo Serra, 237

14025-520 Ribeirão Preto, SP

(c) Sociedade Brasileira de Anestesiologia, 2001
}

\section{SUMMARY}

Stocche RM, Garcia LV, Klamt JG - Effects of Analgesic Intrathecal Sufentanil and $0.25 \%$ Epidural Bupivacaine on Oxytocin and Cortisol Plasma Concentration in Labor Patients

Background and Objectives - Intrathecal sufentanil provides analgesia comparable to epidural local anesthetics for labor pain. Both techniques decrease some parameters of neuroendocrine response to labor pain and both may mitigate reflex oxytocin release in animals. This study aimed to compare the effect of both techniques on cortisol (CPC) and oxytocin $(O P C)$ plasma concentrations in labor patients.

Methods - Participated in this randomized covered study 30 healthy parturients in spontaneous labor with $4-7 \mathrm{~cm}$ cervical dilatation. Group $S$ patients received intrathecal sufentanil (10 $\mu \mathrm{g}$ ) and group B received epidural $0.25 \%$ bupivacaine. Analgesia was assessed by a visual analogue scale and blood oxytocin and cortisol samples were collected before, 30 and 60 minutes after drug administration. Plasma cortisol and oxytocin concentrations were determined by radioimmunoassay.

Results - Group $S$ analgesia was more intense at 30 minutes. $O P C$ and CPC before analgesia were similar for both groups. Group $S$ showed a significant decrease in OPC at 60 minutes and in CPC at 30 and 60 minutes $(p<0.05)$, while for Group $B$ such values remained stable throughout the study.

Conclusions - In the conditions of this study, epidural bupivacaine analgesia was associated to stable plasma cortisol and oxytocin concentrations. Conversely, intrathecal sufentanil promoted a more intense analgesia and decreased $O P C$ and $C P C$.

KEY WORDS - ANALGESIA, labor; ANALGESICS, Opioids: sufentanil; ANESTHETIC TECHNIQUES, Regional: epidural; HORMONES: cortisol, oxytocin

\section{INTRODUÇÃO}

O trabalho de parto induz resposta neuroendócrina e metabólica muito semelhante ao trauma cirúrgico. Provoca aumento dos níveis de catecolaminas ${ }^{1}$, cortisole $\mathrm{ACTH}^{2}$, hormônio liberador de corticotrofina (CFH) e $\beta$-endorfinas ${ }^{3}$. A indução de estresse agudo em macacas grávidas produz diminuição do fluxo útero-placentário e conseqüente hipóxia, hipotensão e bradicardia fetal ${ }^{4}$. O estresse agudo exacerbado também pode provocar retardo na lactogênese e acidose fetal transitória, a qual apresenta correlação com a concentração plasmática do cortisol materno. ${ }^{5,6}$ Assim, o estudo das concentrações plasmáticas de cortisol pode ser 
utilizado como medida desta resposta. O efeito e a importância da ocitocina durante o trabalho de parto estão bem estabelecidos. A ocitocina também está envolvida na lactação em mamíferos ${ }^{7}$ e na resposta ao estresse ${ }^{8}$.

A analgesia de parto com anestésicos locais, por via peridural, possibilita controle da dor, com conseqüente diminuição das concentrações plasmáticas de cortisol, sem alterar a contratilidade uterina ${ }^{9}$. Entretanto a influência desta técnica na concentração plasmática de ocitocina permanece controversa $^{10,11}$. Com o surgimento da técnica raqui-peridural combinadas, a utilização de opióides por via subaracnóidea ficou facilitada. Os opióides, por via subaracnóidea, produzem analgesia intensa com latência curta e sem bloqueio motor. Estudos realizados em animais demonstraram que os opióides inibem a liberação de ocitocina no eixo hipotálamo-hipofisário e, em humanos, a morfina por via venosa pode inibir o trabalho de parto ${ }^{12,13}$. O efeito da analgesia de parto, com opióides por via subaracnóidea, nos níveis plasmáticos de cortisol e ocitocina, ainda não foi relatado.

Os objetivos deste estudo foram verificar e comparar os efeitos da analgesia peridural com bupivacaína a $0,25 \%$, com os efeitos da analgesia com sufentanil por via subaracnóidea no tocante à concentração de cortisol e ocitocina em gestantes com dor intensa durante a fase ativa do primeiro estágio do trabalho de parto.

\section{MÉTODO}

O estudo prospectivo, aleatório e encoberto foi realizado no Hospital das Clínicas de Ribeirão Preto e Maternidade MATER, aprovado pelo Comitê de Ética em Pesquisa da instituição e com consentimento livre e esclarecido das pacientes.

Foram incluídas no estudo 30 pacientes com idades entre 18 e 35 anos, peso entre 50 e $90 \mathrm{~kg}$, estado físico ASA I, primigestas ou secundigestas com cesariana anterior na primeira gestação (primigestas funcionais), na fase ativa do primeiro estágio do trabalho de parto (dilatação cervical $4-7 \mathrm{~cm}$ ) e com o feto encaixado na pélvis. Não foram incluídas no estudo as pacientes com gestação pré ou pós-termo, as que apresentavam sinais de sofrimento fetal agudo ou crônico e as que tivessem recebido ocitocina exógena a menos de uma hora. Os dados de uma paciente, que evoluiu rapidamente para o parto antes da conclusão do estudo (60 minutos), foram excluídos e substituídos na amostra.

\section{Distribuição dos Grupos e Técnica Analgésica}

Quando as pacientes solicitaram analgesia (dor com EAV maior que 7) foram aleatoriamente distribuídas, por meio de sorteio simples, em um dos dois grupos, segundo a técnica analgésica utilizada. Dez minutos antes do bloqueio, todas as pacientes receberam hidratação venosa com $300 \mathrm{ml}$ de solução de Ringer com lactato. No grupo $S(n=15)$, foi realizada punção com conjunto raqui-peridural combinadas com agulhas Touhy $17 \mathrm{G}$ e ponta de lápis $27 \mathrm{G}$ e injetado $2 \mathrm{ml}$ de so- lução contendo $10 \mu \mathrm{g}$ de sufentanil no espaço subaracnóideo. No grupo $B(n=15)$, foi realizada punção peridural com agulha do tipo Touhy $17 \mathrm{G}$ e injetado $12 \mathrm{ml}$ de bupivacaína a $0,25 \%$. Todas as punções foram realizadas no espaço $L_{3}-L_{4}$, com as pacientes na posição sentada. Logo após a administração das drogas e fixação do cateter peridural, as pacientes foram colocadas em decúbito lateral esquerdo com céfaloaclive de 15 a $20^{\circ}$. Finda a coleta de dados, após 60 minutos, todas as pacientes receberam bupivacaína por via peridural, conforme a necessidade, até a finalização do parto.

\section{Monitorização e Coleta de Dados}

Todas as pacientes foram monitorizadas continuamente com cardioscópio, oxímetro de pulso e tiveram medidas as freqüências respiratórias e pressões arteriais (método oscilométrico), antes do bloqueio, a cada dois minutos durante 20 minutos após o bloqueio e, a partir de então, a cada $15 \mathrm{mi}-$ nutos. Durante os sessenta minutos de estudo, somente foram permitidos exames ginecológicos breves e indolores para verificação da dilatação uterina. Em nenhum caso houve ruptura espontânea ou proposital de membranas amnióticas durante o período do estudo.

Aintensidade da dor foi estimada com o auxílio de uma escala analógica visual linear (EAV) de $10 \mathrm{~cm}$, na qual o ponto zero é ausência de dor e o ponto 10 a pior dor possível ou imaginável. As avaliações foram realizadas imediatamente antes do bloqueio e 30 e 60 minutos após. Anotou-se, também, a incidência de efeitos colaterais, tais como náusea, vômitos, sonolência e hipotensão arterial (PAS menor que $90 \mathrm{mmHg}$ ), bem como a necessidade de tratamentos específicos.

\section{Coleta das Amostras Sangüíneas e Dosagem Hormonal}

Em cada momento do estudo (0,30 e 60 minutos), foram colhidas amostras de $8 \mathrm{ml}$ de sangue venoso em seringas plásticas heparinizadas por meio de cateter de teflon posicionado em veia do braço contralateral ao da infusão de líquidos e medicamentos, totalizando $24 \mathrm{ml}$ colhidos ao longo do experimento. Após a coleta, o sangue foi distribuído em dois tubos de ensaio. Um para dosagem do cortisol e outro contendo EDTA $(10 \mu \mathrm{l} / \mathrm{ml}$ de sangue) para dosagem da ocitocina. Os tubos de ensaios foram, temporariamente, estocados em caixa de isopor a temperatura menor que $4^{\circ} \mathrm{C}$, até a finalização do parto. Finda a coleta, as amostras de sangue foram centrifugadas e o plasma obtido foi estocado por período de três meses em refrigerador a $60^{\circ} \mathrm{C}$ até a dosagem dos hormônios.

As dosagens de ocitocina foram realizadas pelo método de radioimunoensaio com extração plasmática prévia. Os erros intra-ensaio e inter-ensaio foram, respectivamente, 2,5\% e $14,1 \%$ ao nível de P0 e ED 50 da curva padrão.

As dosagens de cortisol, também, foram realizadas pelo método de radioimunoensaio, em duplicata sem extração prévia

${ }^{14}$. Os erros intra-ensaio e inter-ensaio foram, respectivamente, $3,5 \%$ e $14 \%$ ao nível de P0 e ED 50 da curva padrão. 


\section{Análise Estatística}

Foram utilizados como controle as medidas hormonais das próprias pacientes, obtidas antes do bloqueio (análise pareada). Para comparação entre os grupos foram calculadas as variações em porcentagens das medidas hormonais iniciais. Os dados estão representados como média \pm EPM. As concentrações hormonais foram analisadas intra-grupo e inter-grupos pelos testes de Friedman (análise de variância pareada e não paramétrico) e Kruskal-Wallis, respectivamente, e variáveis qualitativas ou proporções pelo teste exato de Fisher ou Qui-quadrado. Considerou-se significante valor de $p<0,05$.

\section{RESULTADOS}

Não houve diferenças estatísticas entre os grupos B e S em relação aos resultados demográficos das pacientes (Tabela I). Todas as pacientes se apresentaram normotensas antes do bloqueio (tempo 0). As freqüências cardíaca e respiratória iniciais também foram semelhantes entre os grupos, bem como o grau de paridade e da dilatação cervical (Tabela II). A partir da realização do bloqueio, houve queda transitória da pressão arterial sistólica no Grupo B (30 minutos), com recuperação dos níveis pressóricos aos 60 minutos (análise pareada intra-grupo). No grupo S houve manutenção dos valores pressóricos. Uma paciente do grupo B apresentou pressão arterial sistólica de $90 \mathrm{mmHg}$, que respondeu à infusão rápida de $300 \mathrm{ml}$ de solução de Ringer com lactato. A pressão arterial diastólica e a freqüência cardíaca permaneceram estáveis nos dois grupos durante o período de observação.
Todas as pacientes apresentaram analgesia satisfatória com EAV menor que $3 \mathrm{~cm}$, aos 30 e 60 minutos. Houve diferença entre os grupos nos valores de EAV no tempo 30 minutos (Figura 1).

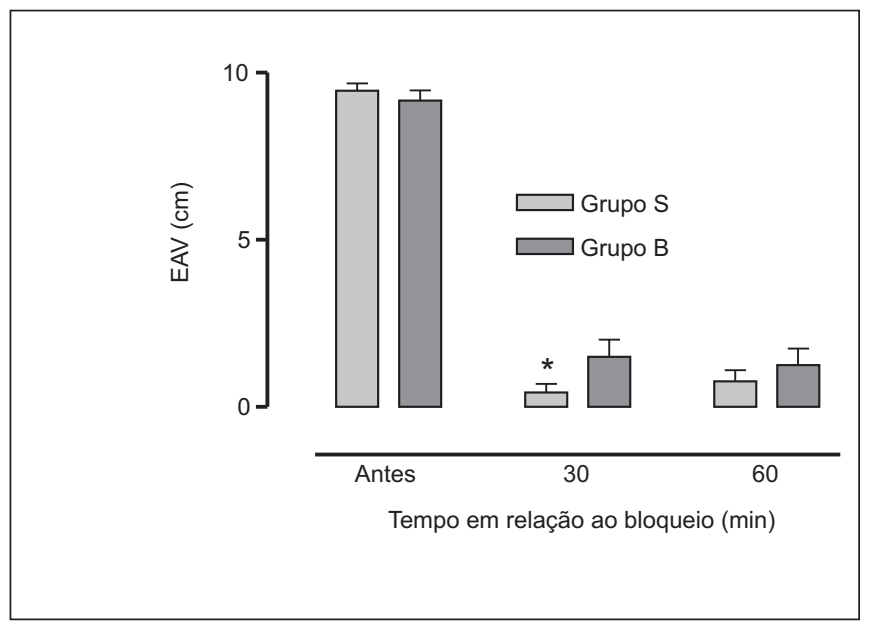

Figura 1 - Intensidade de Dor Avaliada por EAV (cm) para os Grupos S e B, em Relação aos Tempos: antes do Bloqueio, 30 e 60 Minutos após o Bloqueio (Média \pm DP)

* Diferença estatística no tempo 30 minutos, $p<0,05$

As concentrações plasmáticas de ocitocina antes do início da analgesia de parto estiveram elevadas e similares nos dois grupos, apresentando médias $\pm E P M$ de $9,1 \pm 1,1$ no grupo $S$ e $9,8 \pm 1,5 \mathrm{pg} \cdot \mathrm{ml}^{-1}$ no grupo B. Após o bloqueio, ocorreu diminuição dos valores plasmáticos da ocitocina no grupo $S$

Tabela I - Características Demográficas Maternas (Média \pm DP)

\begin{tabular}{lcc}
\hline & $\begin{array}{c}\text { Sufentanil subaracnóideo } \\
10 \mu \mathrm{g}, 2 \mathrm{ml} \\
(\mathrm{n}=15)\end{array}$ & $\begin{array}{c}\text { Bupivacaína peridural } 0,25 \%, \\
12 \mathrm{ml} \\
(\mathrm{n}=15)\end{array}$ \\
\hline Idade (anos) & $22,7 \pm 6,3$ & $22,5 \pm 7,4$ \\
Altura $(\mathrm{cm})$ & $160 \pm 5,2$ & $161 \pm 5,8$ \\
Peso $(\mathrm{kg})$ & $64,4 \pm 9,2$ & $66,8 \pm 11,8$ \\
\hline
\end{tabular}

Tabela II - Parâmetros no Tempo 0 (zero), Utilizados como Controle para os Tempos após o Bloqueio

\begin{tabular}{|c|c|c|}
\hline & $\begin{array}{c}\text { Sufentanil subaracnóideo } \\
10 \mathrm{mg}, 2 \mathrm{ml} \\
(\mathrm{n}=15)\end{array}$ & $\begin{array}{c}\text { Bupivacaina peridural } 0,25 \% \text {, } \\
12 \mathrm{ml} \\
(\mathrm{n}=15)\end{array}$ \\
\hline Nível de dor (EAV) * & $9,5 \pm 0,8$ & $9,1 \pm 1,1$ \\
\hline Dilatação cervical $(\mathrm{cm})$ * & $5,5 \pm 1,7$ & $6,4 \pm 1,6$ \\
\hline \multicolumn{3}{|l|}{ Paridade } \\
\hline 0 & 9 & 10 \\
\hline 1 & 6 & 5 \\
\hline Pressão arterial sistólica $(\mathrm{mmHg})$ * & $126,0 \pm 14,2$ & $129,0 \pm 11,2$ \\
\hline Pressão arterial diastólica $(\mathrm{mmHg})$ * & $80,7 \pm 14,4$ & $86,1 \pm 12,7$ \\
\hline Freqüência cardíaca (bpm) * & $95 \pm 18,3$ & $95 \pm 12,9$ \\
\hline
\end{tabular}

* Dados expressos pela Média \pm DP 
nos tempos 30 e 60 minutos, que apresentou diferença estatística, em análises pareadas com as concentrações antes do bloqueio $(p<0,05)$. O grupo B se comportou de maneira diferente, com manutenção dos valores iniciais durante todo o tempo do estudo (Figura 2).

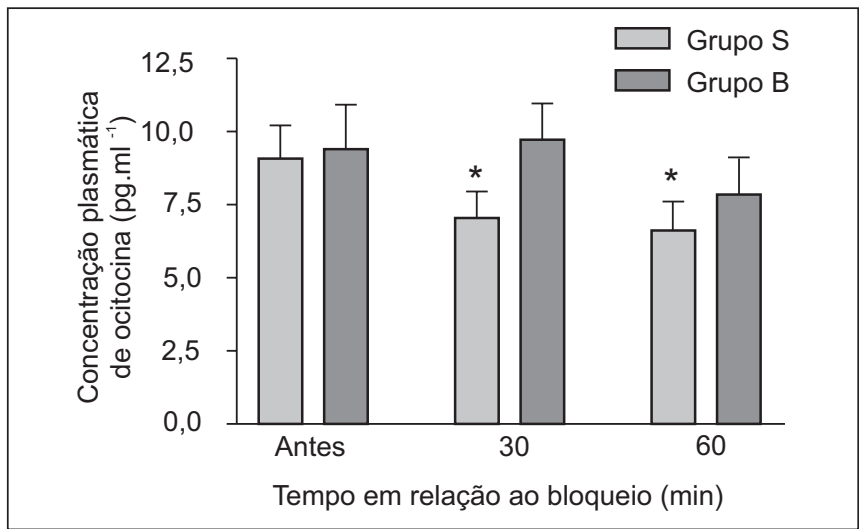

Figura 2 - Concentrações Plasmáticas de Ocitocina (pg.ml $\left.{ }^{-1}\right)$ em Relação ao Tempo de Bloqueio nos Grupos S e B (Média \pm DP) * Diferencas estatísticas intra-grupos, $p<0,05$ (comparacão entre as medidas após bloqueio com a medida antes do bloqueio)

A variação percentual em relação aos valores de ocitocina antes do bloqueio foi utilizada para comparações inter-grupos. Houve variações percentuais negativas no grupo $S$ enquanto ocorreram variações percentuais positivas no grupo $B$, apresentando diferença estatística no tempo $60(p<0,05)$. Apesar das diminuições percentuais de ocitocina no tempo 30 no grupo S, não houve diferença estatística entre os grupos devido às grandes variações no grupo B (Figura 3).

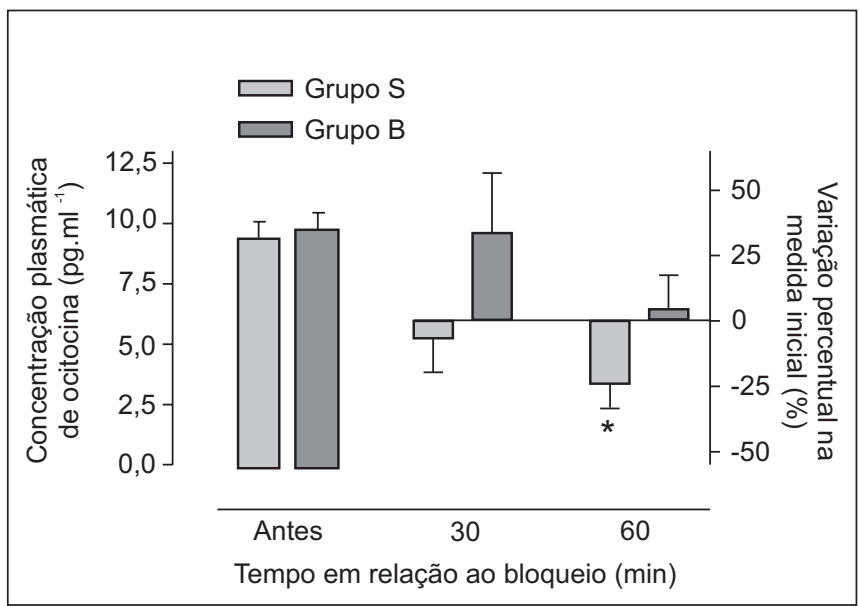

Figura 3 - Médias \pm EPM das Concentrações de Ocitocina antes do Bloqueio (eixo $X$ da esquerda) e Variações em Percentual da Inicial para os Tempos 30 e 60 Minutos pós Bloqueio (eixo X da direita), para os Grupos S e B (Média \pm DP)

* Diferença estatística inter-grupos no tempo 60 minutos, $(\mathrm{p}<0,05)$
As concentrações plasmáticas de cortisol iniciais foram semelhantes nos grupos $S$ e B, com médias \pm DP de 47,8 $\pm 3,6$ e $50,5 \pm 2,8 \mathrm{pg} \cdot \mathrm{ml}^{-1}$, respectivamente. Após o bloqueio, ocorreu diminuição nos valores plasmáticos do cortisol no grupo S no tempo 60, que apresentou significância estatística em análise pareada com a concentração antes do bloqueio ( $p$ $<0,05)$. No grupo $B$, as concentrações plasmáticas permaneceram estáveis (Figura 4).

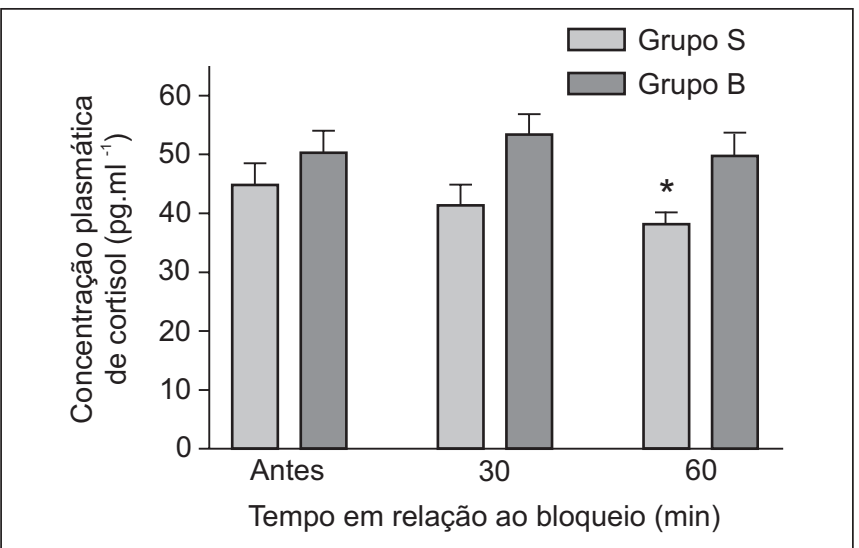

Figura 4 - Concentrações Plasmáticas de Cortisol (pg.ml $\left.{ }^{-1}\right)$ em Relação ao Tempo de Bloqueio nos Grupos S e B (Média \pm DP) * Diferenças estatísticas intra-grupos, $p<0,05$ (comparação entre as medidas pós bloqueio com a medida antes do bloqueio controle)

As variações percentuais das concentrações iniciais individuais foram usadas para comparações inter-grupos. Houve diferença nas variações percentuais das concentrações iniciais de cortisol entre os grupos $\mathrm{S}$ e B nos tempos 30 e $60 \mathrm{mi}-$ nutos $(p<0,05)$ (Figura 5).

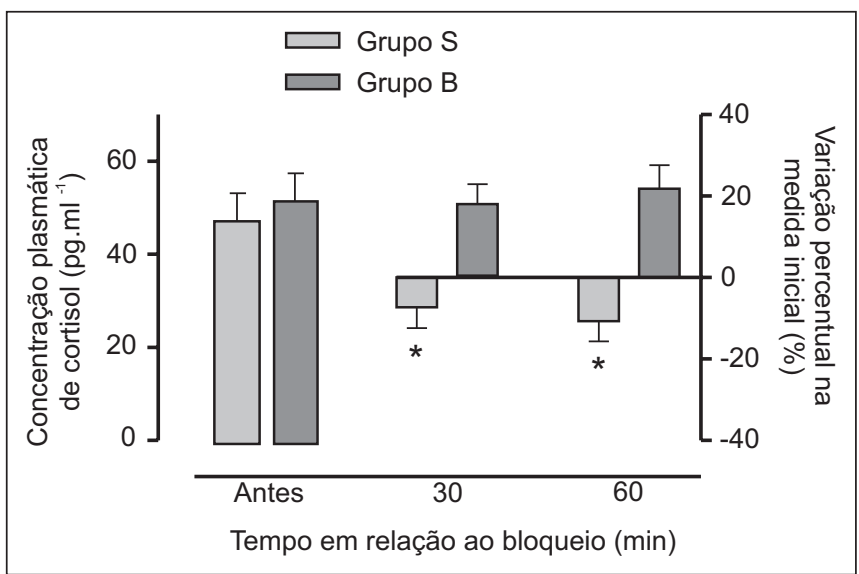

Figura 5 - Concentrações de Cortisol antes do Bloqueio (eixo X da esquerda) e Variações em Percentual da Inicial para os Tempos 30 e 60 Minutos pós Bloqueio (eixo $X$ da direita), para os Grupos S e B (Média \pm DP)

* Diferença estatística inter-grupos nos tempos 30 e 60 minutos, $(p<0,05)$

Revista Brasileira de Anestesiologia Vol. 51, № 4, Julho - Agosto, 2001 
A totalidade das pacientes do grupo S apresentou prurido de leve a moderado, sem necessidade de administração de naloxona. Dez pacientes do grupo S $(66,6 \%)$ apresentaram sonolência leve ou moderada e no grupo B não houve ocorrência de prurido e 2 pacientes $(16,6 \%)$ apresentaram sonolência. Não houve caso de diminuição da saturação de oxigênio abaixo de $92 \%$. Todavia, duas pacientes do grupo S apresentaram bradipnéia transitória com freqüência respiratória de 9 ir/min. Nestes casos não houve necessidade de intervenção, pois as duas pacientes se apresentavam dormindo naquele momento e, logo que foram despertadas, aumentaram a freqüência respiratória.

\section{DISCUSSÃO}

Apesar deste estudo não ser encoberto, a distribuição das pacientes nos grupos S e B foi homogênea. Os valores hormonais observados antes da analgesia apresentaram grande importância neste estudo, pois serviram como controle em análises pareadas com os valores obtidos posteriormente ao bloqueio. Este método permite uma análise fidedigna das alterações proporcionadas pelas analgesias nas variáveis estudadas.

A hipotensão arterial em obstetrícia pode ser deletéria tanto para a mãe como para o feto (diminui a perfusão uterina), podendo produzir diminuição na atividade uterina e hipóxia fetal $^{15}$. Ensaios clínicos demonstraram que tanto a analgesia com sufentanil por via subaracnóidea quanto a analgesia peridural produzem diminuição semelhante da pressão arterial 16,17 . Nossos resultados diferem dos encontrados nestes ensaios, pois as variações da pressão arterial sistólica (PAS) em relação ao controle (medida no tempo zero) foram maiores no grupo B, no tempo 30 minutos. Entretanto, nestes estudos, não há descrição de que as pacientes tenham recebido expansão volumétrica antes do bloqueio, levando à maior probabilidade de hipotensão arterial, o que difere do nosso método. Em nosso estudo, uma paciente do grupo B apresentou diminuição da PAS abaixo de $90 \mathrm{mmHg}$ que respondeu a infusão de solução de Ringer com lactato, sugerindo que houve alterações hemodinâmicas mais intensas neste grupo.

Duas pacientes do grupo $S$ apresentaram freqüência respiratória de $9 \mathrm{irpm}$, no entanto, nenhuma apresentou $\mathrm{SpO}_{2}$ inferior a $92 \%$. A sonolência após a instalação da analgesia pode ser explicada pela atenuação do estresse e fadiga materna. Entretanto, a maior incidência de sonolência no grupo $S(66,6 \%)$ contra $16,6 \%$ no grupo $B$, bem como todas pacientes do grupo $S$ terem apresentado prurido sugerem dispersão rostral da droga. Estudo da farmacocinética do sufentanil em ovelhas demonstrou que a administração subaracnóidea é seguida de concentrações elevadas da droga na cisterna magna após 10 minutos e que as concentrações liquóricas se apresentaram sempre mais elevadas do que as plasmáticas, comprovando a dispersão rostral por via liquórica ${ }^{18}$. A baricidade da solução contendo opióide pode influenciar a dispersão rostral e a eficácia da analgesia ${ }^{19}$ e o sufentanil na apresentação $5 \mu \mathrm{g} \cdot \mathrm{ml}^{-1}$ mostra-se hipobárico a $37^{\circ} \mathrm{C}$, o que favorece a dispersão cefálica se a paciente permanecer maior tempo sentada durante a realização do bloqueio ${ }^{20}$. Em nosso estudo, o tempo necessário para as passagens de catéteres peridurais após as injeções subaracnóideas foi inferior a 5 minutos.

A latência da analgesia de parto com sufentanil por via subaracnóidea é menor do que a latência da peridural com anestésico local ${ }^{17}$. Em nosso estudo reproduzimos estes resultados, onde ocorreu diferença estatística na intensidade de dor até 30 minutos após o início da analgesia.

É consensual o fato de que a concentração plasmática de cortisol aumenta progressivamente durante a gestação, mantendo o ritmo circadiano, chegando no final da gravidez com titulação substancialmente maior do que nas mulheres não grávidas ${ }^{2}$. Após o início do trabalho de parto existe um aumento progressivo do cortisol, com pico de concentração plasmática logo após o nascimento ${ }^{21}$. Esta elevação é parcialmente inibida pela analgesia de parto peridural com anestésicos locais ${ }^{9}$.

O cortisol pode influenciar o trabalho de parto, pois, no final da gravidez, aumenta a expressão do RNAmensageiro do receptor de ocitocina em ovelhas, preparando o miométrio para o trabalho de parto ${ }^{22}$. O fato do cortisol diminuir a produção de progesterona e aumentar a de estrogênio, via ativação da $17 \alpha$-hidroxilase ${ }^{23}$, e inibir a 15-hidroxiprostaglandina desidrogenase ${ }^{24}$ sugere um papel importante do cortisol no desencadeamento do trabalho de parto. No entanto, o aumento durante o trabalho de parto está diretamente ligado ao estresse emocional e intensidade da dor e parece estar relacionado ao surgimento de infecção e alterações psíquicas pós-parto ${ }^{25,26}$.

Nossos resultados confirmaram concentrações plasmáticas elevadas de cortisol na fase ativa do trabalho de parto e diminuição após a realização da analgesia no grupo S que diferiu do grupo $B$, que se manteve constante. Isto provavelmente se deveu à presença mais freqüente de dor residual no grupo B.

Vários autores tentaram correlacionar o bloqueio da dor com a atenuação da resposta neuroendócrina ${ }^{27}$. No entanto, nestes estudos, não foi possível eliminar a possibilidade da participação do bloqueio de outras vias neurais que não as da dor. Em nosso estudo, o bloqueio marcante na liberação de cortisol no grupo S sugere que as vias de dor têm papel importante na resposta neuroendócrina. Existe, também, a possibilidade de dispersão cefálica do opióide com inibição da liberação de fator liberador de corticotrofina ${ }^{28}$. Assim, o sufentanil por via subaracnóidea em analgesia de parto produz bloqueio da liberação do cortisol pelo eixo hipotálamo-hipofisário, provavelmente por bloqueio da dor, sugerindo importante participação das vias da dor na resposta neuroendócrina ao estresse.

Os estudos que envolvem dosagem de ocitocina em trabalho de parto apresentam algumas dificuldades metodológicas. Devido à sua rápida metabolização via ocitocinase, a concentração no sangue venoso periférico representa uma fração da encontrada na veia jugular interna. A liberação em pulsos da ocitocina na corrente sangüínea é outro fator que 
pode influenciar os níveis de ocitocina plasmática periférica ${ }^{29}$. Por isso, em nosso estudo, não permitimos manobras obstétricas e ruptura da membrana amniótica durante os 60 minutos do estudo, e as coletas das amostras sangüíneas foram realizadas sempre durante o pico de intensidade da contração uterina. Outro fator que dificulta a interpretação dos resultados é a ampla variação da concentração plasmática de ocitocina entre as pacientes em trabalho de parto. Assim, usamos uma dosagem imediatamente antes da analgesia como controle para cada paciente analisada, seguindo o método aplicado em outros estudos ${ }^{30}$.

Poder-se-ia esperar que o uso de sufentanil por via subaracnóidea não interferisse no reflexo de Ferguson por agir, especificamente, na coluna dorsal da medula espinal, modulando, preferencialmente, as vias da dor, sem influenciar as vias de sensibilidade de distensão do períneo. No entanto, nossos dados mostram que a ocitocina plasmática reduziu-se, significativamente, após a administração de sufentanil por via subaracnóidea em relação ao controle, o que não ocorreu com o uso de anestésico local peridural. Esta diminuição da ocitocina demonstra a interferência de um opióide $\mu$ específico, potente e de rápida ação, administrado por via subaracnóidea, no reflexo de Ferguson, o que poderia influenciar diretamente a evolução do trabalho de parto.

Duas hipóteses poderiam explicar a diminuição da ocitocina plasmática no grupo $S$ e não no grupo $B$ em nosso estudo. $A$ primeira é de que o reflexo de Ferguson apresenta forte aferência de vias de dor, o que justificaria a manutenção da concentração plasmática da ocitocina no grupo $B$, pois este apresentou analgesia adequada, porém menos profunda que no grupo S. Nesta hipótese, a ação sobre a ocitocina, tanto dos opióides quanto dos anestésicos locais, estariam preferencialmente na medula espinal. A segunda hipótese é que o sufentanil por via subaracnóidea apresenta dispersão rostral, atingindo a região do eixo hipotálamo-hipofisário, inibindo a liberação de ocitocina pelo lobo posterior da hipófise ao nível central.

Os mecanismos endógenos de controle da secreção de ocitocina são de alta complexidade e não foram totalmente esclarecidos. A ação dos opióides no eixo hipotálamo-hipofisário $(\mathrm{EHH})$, inibindo a liberação de ocitocina, foi descrita em estudos experimentais in vitro ${ }^{31}$ e in vivo em camundongos ${ }^{32}$.

A presença e ação de receptores opióides $\mu \mathrm{e} \kappa$ no eixo hipotálamo-hipofisário foi amplamente demonstrada, especialmente nos Núcleos Supra-Ópticos e Paraventriculares ${ }^{33}$. Nos neurônios magnocelulares dos Núcleos Supra-Ópticos, a população de receptores $\kappa$ é maior que a $\mu$ e sua ativação promove a diminuição da atividade elétrica e hiperpolarização neuronal, inibindo a liberação de ocitocina pela neuro-hipófise ${ }^{34}$. No entanto, em ratas no final da gestação, observaram-se diminuição da resposta dos agonistas $\kappa$ e aumento da ação dos receptores $\mu^{13,35}$. No lobo posterior da hipófise há evidências da existência de populações de receptores $\mu \mathrm{e} \kappa$, pois a naloxona (antagonista seletivo $\mu$ ) intracerebral promove liberação de ocitocina ${ }^{31}$.

Em ratas, a atividade uterina bem como os valores plasmáticos de ocitocina durante o trabalho de parto diminuíram após o uso de morfina subcutânea ou intracerebral, levando ao prolongamento do trabalho de parto e evidenciando a ação inibidora dos opióides $\mu$ agonistas no reflexo de Ferguson em nível central ${ }^{36}$. Apesar de extensa bibliografia sobre a modulação dos opióides na liberação de ocitocina em animais, poucos estudos foram realizados em humanos, possivelmente pelas dificuldades metodológicas e éticas em realizar tais experimentos. Com o desenvolvimento e grande expansão da técnica de analgesia subaracnóidea com opióides lipossolúveis no final da década de 80 , este tema passou a apresentar grande importância.

A morfina, por via venosa, na dose de $5 \mathrm{mg}$, administrada na primeira fase do trabalho de parto, diminuiu a ocitocina plasmática na circulação periférica, provavelmente, por inibir a liberação no eixo hipotálamo-hipofisário ${ }^{12}$, estabelecendo a primeira correlação entre os trabalhos clínicos e os experimentais. Na segunda fase do trabalho de parto, a morfina, por via venosa, não foi capaz de bloquear a liberação de ocitocina, provavelmente, devido à maior intensidade do estímulo e à baixa efetividade analgésica da morfina venosa em baixas doses nesta fase ${ }^{37}$. A resposta inibitória na liberação de ocitocina devido ao trabalho de parto, tanto dos agonistas $\kappa$ quanto dos agonistas $\mu$, depende do número e intensidade das aferências sensitivas ${ }^{38}$, o que fortalece a possibilidade da inibição da liberação de ocitocina por opióides depender do estágio do trabalho de parto.

A possibilidade de dispersão cefálica do sufentanil já foi discutida anteriormente. Não pudemos demonstrar que o sufentanil ascendeu até a região supraespinal, mas a alta incidência de sonolência e prurido e a diminuição da freqüência respiratória em duas pacientes do grupo S sugerem a possibilidade de dispersão rostral. O fato de a analgesia não alterar a ocitocina plasmática no grupo B sugere que outros mecanismos, que não o bloqueio da dor, estejam envolvidos na diminuição da ocitocina no grupo S e que o local da ação do sufentanil, diminuindo os níveis de ocitocina, deve ser supraespinal.

Estudos prévios do efeito da analgesia peridural com anestésicos locais na concentração plasmática de ocitocina obtiveram resultados e conclusões distintas. Pacientes que receberam analgesia peridural tiveram diminuição da taxa de ocitocina plasmática após o bloqueio ${ }^{10}$, diferindo dos nossos resultados. Nesse estudo, porém, a analgesia foi realizada no final do segundo estágio do trabalho de parto em que a aferência sensitiva e conseqüente liberação da ocitocina é intensa e o bloqueio completo com anestésico local poderia apresentar maior efeito na liberação reflexa da ocitocina, diferindo do nosso método. Apesar de não ter havido diminuição da ocitocina no grupo B de nossa amostra, também não houve elevação, como era de se esperar durante a evolução natural do trabalho de parto.

A técnica de analgesia de parto com opióides por via subaracnóidea não produz perda da prensa abdominal e não relaxa a musculatura da pelve. Tais fatores poderiam causar distócia de rotação e prolongar o trabalho de parto, o que poderia explicar a evolução do trabalho de parto mais rápida com esta técnica. Apesar da aparente manutenção do trabalho de Vol. 51, N 4, Julho - Agosto, 2001 
parto, os efeitos da diminuição da taxa de ocitocina na contratilidade uterina e a evolução do trabalho de parto não foram objetivos de nosso estudo. Os mecanismos de controle da contratilidade uterina são complexos e parcialmente elucidados. Outros fatores como diminuição nas concentrações plasmáticas de catecolaminas e o número de receptores de ocitocina no miométrio podem contrabalancear a diminuição da ocitocina.

Concluindo, a administração subaracnóidea de sufentanil produz diminuição das concentrações plasmáticas de ocitocina plasmática. O provável mecanismo de ação é a inibição da liberação no eixo hipotálamo-hipofisário. A comprovação do mecanismo de ação e a influência na contratilidade uterina necessitam de estudos adicionais, com método específico.

\section{Effects of Analgesic Intrathecal Sufentanil and $0.25 \%$ Epidural Bupivacaine on Oxytocin and Cortisol Plasma Concentration in Labor Patients}

Renato Mestriner Stocche, M.D., Luiz Vicente Garcia, M.D., Jyrson Guilherme Klamt, M.D.

\section{INTRODUCTION}

Labor induces a neuroendocrine and metabolic response very similar to surgical trauma. It increases the levels of catecholamine ${ }^{1}$, cortisol and $A C T H^{2}$, corticotrophin (CFG) releasing hormones and $ß$-endorphins ${ }^{3}$. Induced acute stress in pregnant monkeys decreased uterus-placental flow leading, as a consequence, to fetal hypoxia, hypotension and bradycardia ${ }^{4}$. Exacerbated acute stress may also cause lactogenesis delay and transient fetal acidosis, which has a correlation with maternal cortisol plasma concentrations ${ }^{5,6}$. So, cortisol plasma concentrations may be used as a measure of such response. Oxytocin effect and importance during labor are well established. Oxytocin is also involved in lactation ${ }^{7}$ and stress response ${ }^{8}$.

Labor analgesia with epidural local anesthetics allows for pain control with a consequent decrease in cortisol plasma levels, however without changing uterine contractility ${ }^{9}$. Nevertheless, the influence of such technique in oxytocin plasma concentrations remains controversial ${ }^{10,11}$. The combined spinal-epidural technique has made easier the use of epidural opioids. Intrathecal opioids produce intense analgesia with fast onset and without motor block. Animal studies have shown that opioids inhibit oxytocin release in the hypothalamohypophyseal axle and, in humans, intravenous morphine may inhibit labor ${ }^{12,13}$. The effects of labor analgesia with intrathecal opioids on cortisol and oxytocin plasma concentrations has not been reported to date.
This study aimed to evaluate and compare the effects of epidural analgesia with $0.25 \%$ bupivacaine and with intrathecal sufentanil as to cortisol and oxytocin concentrations in pregnant women with intense labor pain during the active phase of the first labor stage.

\section{METHODS}

This prospective, randomized and blind study was performed in Hospital das Clinicas, Ribeirão Preto and Maternidade MATER after the Institution's Research Ethics Committee approval and the informed consent of patients.

Participated in this study 30 patients aged 18 to 35 years, weighing 50 to $90 \mathrm{~kg}$, physical status ASAI, primiparous or on second gestation with Cesarean section in the first gestation (functional primiparous), in the active phase of the first labor stage (cervical dilatation $=4-7 \mathrm{~cm}$ ) and with the fetus fit in the pelvis. Patients with pre or post term gestation, presenting signs of acute or chronic fetal suffering and those having received exogenous oxytocin less than one hour before were excluded from the study. Data of one patient who quickly evolved to delivery before the end of the study (60 minutes) were excluded and replaced in the sample.

\section{Groups Distribution and Analgesic Technique}

When patients asked for analgesia (VAS pain above 7), they were randomly distributed into one of two groups according to the analgesic technique used. Ten minutes before blockade, all patients received venous hydration with $300 \mathrm{ml}$ lactated Ringer's. Puncture was performed in Group $S(n=15)$ with a combined spinal-epidural set with $17 \mathrm{G}$ Tuhoy and $27 \mathrm{G}$ pencil tip needles and $2 \mathrm{ml}$ solution with $10 \mu \mathrm{g}$ sufentanil was intrathecally injected. Epidural puncture was performed in Group B ( $n=15)$ with $17 \mathrm{G}$ Tuhoy needles and $12 \mathrm{ml}$ of $0.25 \%$ bupivacaine were injected. All punctures were performed at $\mathrm{L}_{3}-\mathrm{L}_{4}$ interspace with patients in the sitting position. Immediately after drug administration and epidural catheter fixation, patients were placed in the left lateral position with heads up $15^{\circ}$ to $20^{\circ}$. After data collection, 60 minutes after, all patients received epidural bupivacaine as needed until delivery.

\section{Monitoring and Data Collection}

All patients were continuously monitored with cardioscope, pulse oximetry and respiratory frequency and blood pressure (oscilloscope) were measured before blockade, at every two minutes for 20 minutes after blockade and after this, at every 15 minutes. During this sixty-minute study, only brief and painless gynecologic exams were allowed to determine uterine dilatation. There has been no spontaneous or provoked membrane rupture during the study.

Pain intensity was evaluated with the help of a $10 \mathrm{~cm}$ linear visual analog pain scale (VAS) where zero was lack of pain and 10 the worst possible or imaginable pain. Evaluations were performed immediately before the blockade and 30 and 60 minutes after it. The incidence of side-effects, such as nau- 
sea, vomiting, somnolence and hypotension (SBP below 90 $\mathrm{mmHg}$ ) were also recorded, as well as the need for specific treatments.

\section{Blood Sample Collection and Hormone Dosage}

Venous blood samples $(8 \mathrm{ml})$ were collected with plastic heparin-containing syringes through a teflon catheter placed in a vein of the arm opposite to that receiving fluids and drugs at all study moments ( 0,30 and 60 minutes), in a total of $24 \mathrm{ml}$ collected along the experiment. After collection blood was distributed in two test tubes, one for cortisol dosage and the other containing EDTA $(10 \mu \mathrm{l} / \mathrm{ml}$ blood $)$ for oxytocin dosage. Test tubes were then temporarily stored in a styrofoam box at a temperature below $4{ }^{\circ} \mathrm{C}$, until delivery. After collection, samples were centrifuged and plasma was stored for three months at $60{ }^{\circ} \mathrm{C}$ until hormonal dosage.

Oxytocin dosages were obtained by radioimmunoassay with previous plasma extraction. Intra and inter-assay errors were $2.5 \%$ and $14.1 \%$ respectively at the level of PO and ED 50 of the standard curve.

Cortisol dosages were also performed by radioimmunoassay in duplication without previous extraction ${ }^{14}$. Intra and inter-assay errors were $3.5 \%$ and $14 \%$ respectively at the level of P0 and ED 50 of the standard curve.

\section{Statistical Analysis}

Hormonal measurements of patients were used as control and obtained before blockade (paired analysis). To compare groups, initial hormonal measurement variations were calculated. Data are shown in mean \pm EPM. Hormonal concentrations were analyzed intra and inter groups by Friedman (paired and non-parametric analysis of variance) and Kruskal-Wallis tests, respectively. Qualitative variables or proportions were analyzed by Fisher's Exact or Chi-square test. $\mathrm{P}<0.05$ was considered statistically significant.

\section{RESULTS}

There were no statistical differences between groups as to demographics data (Table I). All patients were normotensive before blockade (time 0). Baseline heart and respiratory rates were also similar for both groups, as well as the degree of parity and cervical dilatation (Table II). After blockade there has been a transient decrease in systolic blood pressure in Group B (30 minutes) with pressure levels recovery at 60 minutes. One group B patient presented with systolic blood pressure of $90 \mathrm{mmHg}$, which responded to a fast infusion of $300 \mathrm{ml}$ lactated Ringer's. Diastolic blood pressure and heart rate remained stable for both groups during the observation period.

All patients had satisfactory analgesia with AVS below $3 \mathrm{~cm}$, at 30 and 60 minutes. There has been difference between groups in AVS values at 30 minutes (Figure 1).

Oxytocin plasma concentrations before labor analgesia were increased and similar for both groups, with means \pm

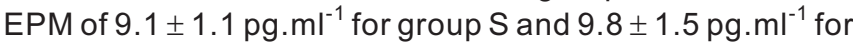
group B. After blockade, there has been a decrease in oxytocin plasma levels in group $S$ at 30 and 60 minutes, which was statistically significant in paired analysis with pre-blockade concentrations ( $p<0.05)$. Group B behaved differently and maintained baseline values throughout the study (Figure 2).

Table I - Maternal Demographics Characteristics (Mean \pm SD)

\begin{tabular}{lcc} 
& $\begin{array}{c}\text { Intrathecal sufentanil } \\
10 \mathrm{mg}, 2 \mathrm{ml} \\
(\mathrm{n}=15)\end{array}$ & $\begin{array}{c}\text { Epidural bupivacaine } 0.5 \% \\
12 \mathrm{ml} \\
(\mathrm{n}=15)\end{array}$ \\
\hline Age (years) & $22.7 \pm 6.3$ & $22.5 \pm 7.4$ \\
Height $(\mathrm{cm})$ & $160 \pm 5.2$ & $161 \pm 5.8$ \\
Weight $(\mathrm{kg})$ & $64.4 \pm 9.2$ & $66.8 \pm 11.8$ \\
\hline
\end{tabular}

Table II - Parameters at Time 0 (zero) Used as Controls for Times after Blockade

\begin{tabular}{lcc}
\hline & $\begin{array}{c}\text { Intrathecal sufentanil } \\
10 \mathrm{mg}, 2 \mathrm{ml} \\
(\mathrm{n}=15)\end{array}$ & $\begin{array}{c}\text { Epidural bupivacaine } 0.25 \%, \\
12 \mathrm{ml} \\
(\mathrm{n}=15)\end{array}$ \\
\hline Pain level (AVS) ${ }^{*}$ & $9.5 \pm 0.8$ & $9.1 \pm 1.1$ \\
Cervical dilatation $(\mathrm{cm})^{*}$ & $5.5 \pm 1.7$ & $6.4 \pm 1.6$ \\
Parity & & 10 \\
$\quad 0$ & 9 & 5 \\
1 & 6 & $129.0 \pm 11.2$ \\
Systolic blood pressure $(\mathrm{mmHg})^{*}$ & $126.0 \pm 14.2$ & $86.1 \pm 12.7$ \\
Diastolic blood pressure $(\mathrm{mmHg})^{*}$ & $80.7 \pm 14.4$ & $95 \pm 12.9$ \\
Heart rate $(\mathrm{bpm})^{*}$ & $95 \pm 18.3$ & \\
\hline
\end{tabular}

\footnotetext{
* Data expressed in Mean \pm SD
} 


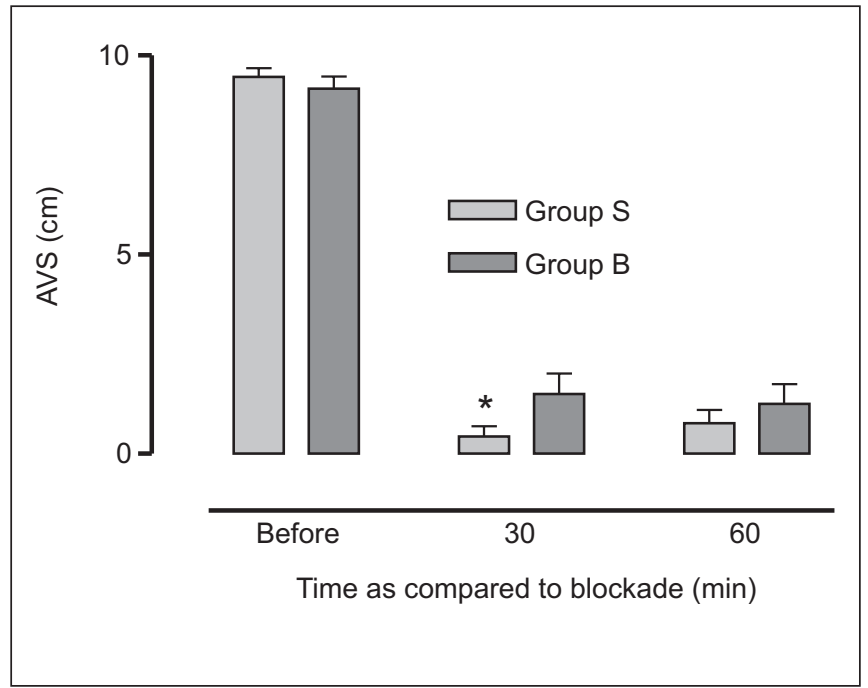

Figure 1 - Pain Intensity Evaluated by AVS (cm) for Groups $S$ and B as compared to Times before Blockade, 30 and 60 Minutes after Blockade (Mean \pm SD)

* Statistical differences at 30 minutes $(p<0.05)$

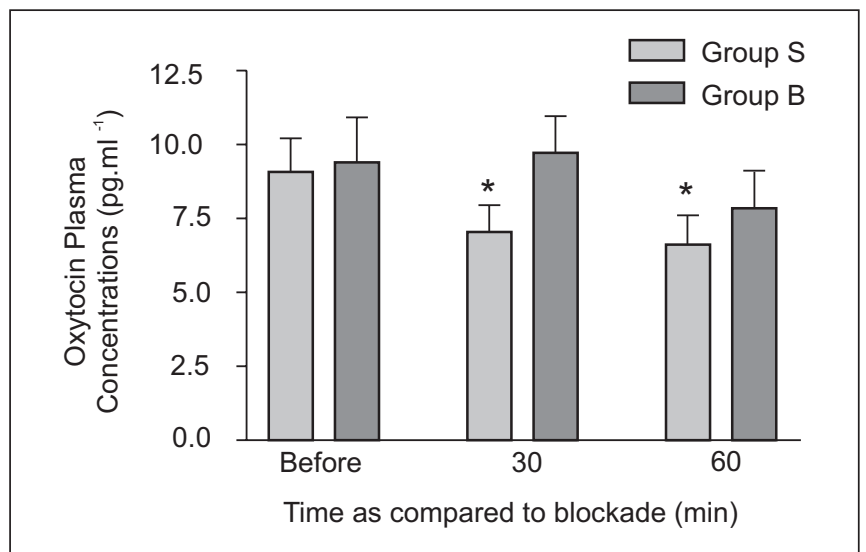

Figure 2 - Oxytocin Plasma Concentrations (pg. $\mathrm{ml}^{-1}$ ) as Compared to Blockade Time in Groups $S$ and $B($ Mean \pm SD)

* Intragroups statistical differences, $p<0.05$ (comparison of measurements after and before blockade

Percentage variation of pre-blockade oxytocin values was used for intergroup comparisons. There were negative percentage variations in group $S$ while there were positive percentage variations in group $B$, with statistical difference at 60 minutes $(p<0.05)$. In spite of percentage oxytocin decreases at moment 30 in group $S$, there has been no statistical difference between groups due to major group $B$ variations (Figure 3).

Initial plasma cortisol concentrations were similar for groups $S$ and $B$, with mean $\pm S D$ of $47.8 \pm 3.6$ and $50.5 \pm 2.8 \mathrm{pg} . \mathrm{ml}^{-1}$, respectively. After blockade, cortisol plasma levels decreased in group $S$ at moment 60 , with statistical difference in paired analysis with pre-blockade concentrations $(p<$ $0.05)$. Plasma concentrations remained stable in group $B$ (Figure 4).

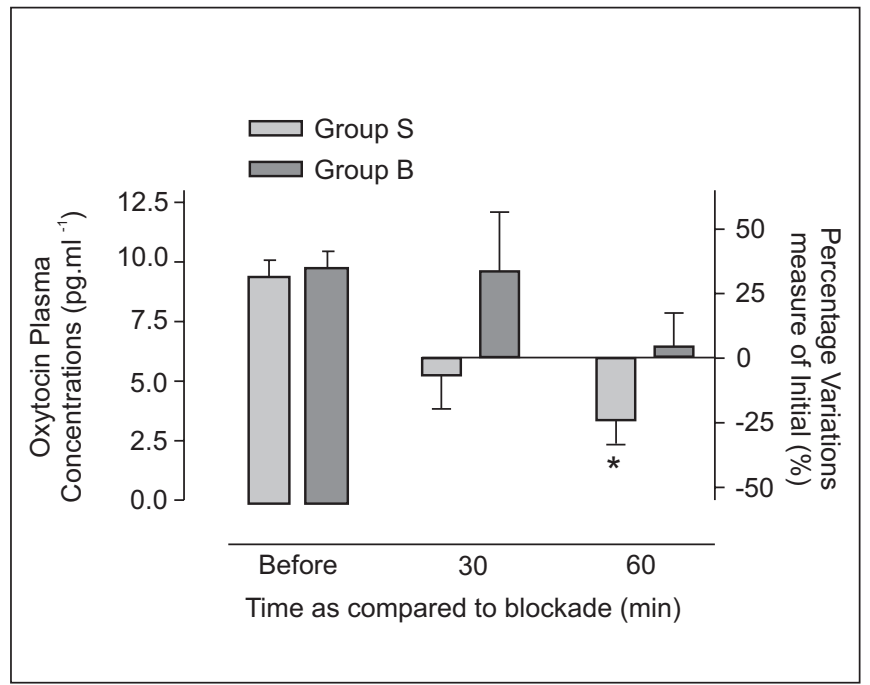

Figure 3 - Mean \pm EPM of Oxytocin Concentrations before Blockade (left $x$ axle) and Perceantage Variations of Initial for Times 30 and 60 Minutes after Blockade (right $x$ axle), for Groups $S$ and $B($ Mean \pm SD)

* Statistical difference intergroups at 60 minutes $(p<0.05)$

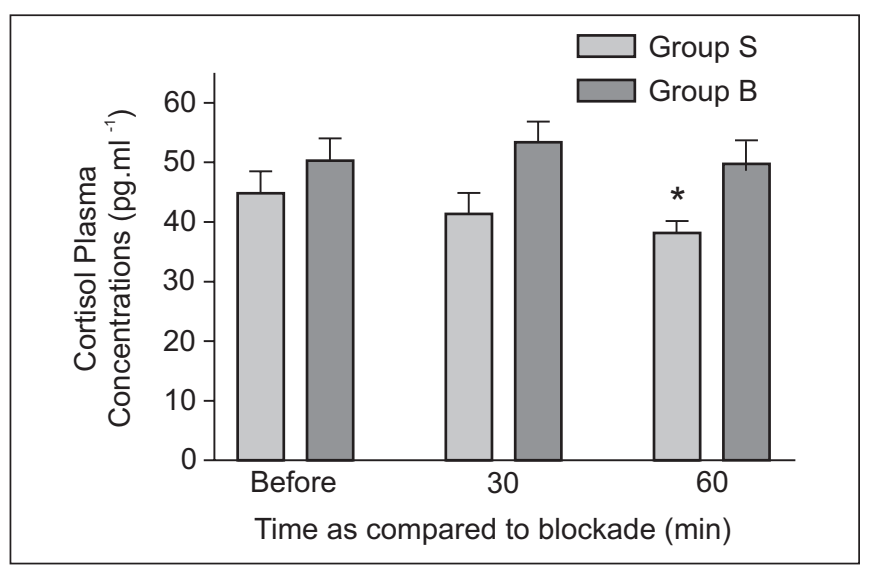

Figure 4 - Cortisol Plasma Concentrations (pg. ml ${ }^{-1}$ ) Compared to Blockade Time in Groups S and B (Mean \pm SD)

*Statistical differences intergroups, $p<0.05$ (comparison of post-blockade and pre-blockade (control) measurements)

Percentage variations of initial individual concentrations were used for intergroup comparisons. There were differences in percentage variations of initial cortisol concentrations between groups $S$ and $B$ at 30 and 60 minutes $(p<0.05)$ (Figure 5).

All group $S$ patients presented with mild and moderate pruritus with no need for naloxone administration. Ten group $S$ patients $(66.6 \%)$ presented with mild or moderate somnolence. There has been no pruritus in group $B$ and two patients $(16.6 \%)$ presented with somnolence. There has been no oxygen saturation decrease below $92 \%$. However, two group B patients presented with transient bradypnea with respiratory 


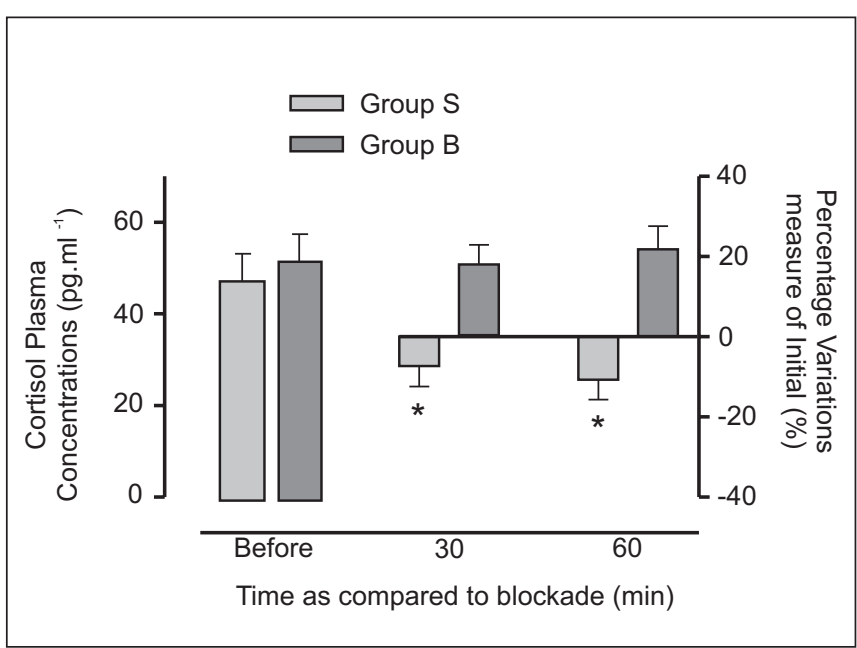

Figure 5 - Pre-Blockade Cortisol Concentrations (left $x$ axle) and Percentage Variations of Initial for Times 30 and 60 Minutes Post-Blockade (right x axle), for Groups $S$ and $B$ (Mean \pm SD)

* Statistical difference intergroups at 30 and 60 minutes $(p<0.05)$

rate of $9 \mathrm{ir} / \mathrm{min}$, but with no need for intervention because both patients were asleep at that time and as soon as awakened their respiratory rate increased.

\section{DISCUSSION}

Although not being blind, this study was homogeneous as to group's distribution. Hormone values before analgesia were especially important for this study because they worked as controls in paired analysis with post-blockade values, thus allowing for an accurate analysis of analgesia-induced changes in all variables studied.

Obstetric hypotension may be noxious both for the mother and the fetus (decreases uterine perfusion), and may decrease uterine activity leading to fetal hypoxia ${ }^{15}$. Clinical studies have shown that both spinal sufentanil and epidural analgesia reduce blood pressure in a similar manner ${ }^{16,17}$. Our results are different from those studies because systolic blood pressure (SBP) variations were higher for group B at 30 minutes, as compared to controls (time zero). However, there are no descriptions in those studies of patients receiving pre-blockade volume expansion, leading to a higher chance of hypotension, what is different from our method. In our study, one group B patient had an SBP decrease below 90 $\mathrm{mmHg}$, which responded to lactated Ringer's infusion, thus suggesting that there were more intense hemodynamic changes in this group.

Two group S patients had a respiratory rate of $9 \mathrm{irpm}$, but none had $\mathrm{SpO}_{2}$ below $92 \%$. Somnolence after analgesia induction may be explained by maternal stress and fatigue attenuation. However, a higher incidence of somnolence in group $S$ $(66.6 \%)$, as compared to $16.6 \%$ in group B, as well as all group $S$ patients presenting with pruritus, suggest a rostral spread of the drug. Pharmacokinetic studies of sufentanil in ewes have shown that intrathecal administration is followed by high drug concentrations in the cisterna magna after 10 minutes and that CSF concentrations are always higher than plasma concentrations, confirming CSF rostral spread ${ }^{18}$. Opioid-containing solutions baricity may influence rostral spread and analgesia efficacy ${ }^{19}$ and $5 \mu \mathrm{g} \cdot \mathrm{kg}^{-1}$ sufentanil is hypobaric at $37^{\circ} \mathrm{C}$, which favors the cephalic spread if the patient remains too long in the sitting position during blockade ${ }^{20}$. In our study, time needed for epidural catheters insertion after intrathecal injections was less than 5 minutes.

Labor analgesia onset with intrathecal sufentanil is faster than epidural onset with local anesthetics ${ }^{17}$. Our study has reproduced such results with statistical differences in pain intensity up to 30 minutes after beginning of analgesia.

It is a consensus that cortisol plasma concentrations progressively increase during gestation, maintaining the circadian rhythm and reaching the end of gestation with a substantially higher titration as compared to non-pregnant women ${ }^{2}$. When labor begins there is a progressive cortisol increase with peak plasma concentration soon after birth ${ }^{21}$. Such increase is partially inhibited by epidural labor analgesia with local anesthetics ${ }^{9}$.

Cortisol may influence labor because, in ewes at the end of gestation, oxytocin receptor messenger RNA expression increased to prepare myometrium for labor ${ }^{22}$. The fact that cortisol decreases progesterone and increases estrogen production via $17 \alpha$-hydroxylase ${ }^{23}$ activation and 15-hydroxyprostaglandin dehydrogenase inhibition ${ }^{24}$ suggests an important cortisol role in triggering labor. However, the increase during labor is directly related to emotional stress and pain intensity and seems to be related to post-partum infection and psychic alterations ${ }^{25-26}$.

Our results confirmed high cortisol plasma concentrations in the active phase of labor, which decreased after analgesia in group $B$ and was different from group $S$ where it remained constant. This was probably due to the higher incidence of residual pain in group $B$.

Several authors tried to correlate pain blockade with neuroendocrine response attenuation ${ }^{27}$. However, in those studies, it was impossible to rule out the participation of neural blockades, other than pain. In our study, the marked cortisol release blockade in group S suggests that pain pathways have an important role in the neuroendocrine response. There is also the possibility of cephalic opioid spread with inhibition of corticotropin releasing factor release ${ }^{28}$. So, intrathecal sufentanil in labor analgesia blocks cortisol release by the hipothalamohypophysial axle probably by blocking pain, suggesting an important participation of pain pathways in neuroendocrine stress response.

Studies involving oxytocin labor dosages have some methodological difficulties. Due to its fast metabolization via oxytocinase, peripheral venous blood concentration is a fraction of that found in the internal jugular vein. Oxytocin pulses release in blood flow is another factor which may influence 
oxytocin peripheral plasma levels ${ }^{29}$. So, we did not allow obstetric maneuvers and membrane rupture during the $60 \mathrm{~min}-$ utes of study, and blood samples were collected always during uterine peak contraction intensity. Another factor impairing results interpretation is the wide variation in oxytocin plasma levels among labor patients. So, we used the dose immediately before analgesia as the control for each patient, according to methods used in other studies ${ }^{30}$.

It was to be expected that intrathecal sufentanil would not interfere with Ferguson's reflex for acting specifically on the dorsal spine of the spinal cord, preferably modulating pain pathways without affecting perineal distension sensitivity pathways. However, our data show that plasma oxytocin was significantly reduced after intrathecal sufentanil administration as compared to control, what was not seen with the use of epidural local anesthetics. This oxytocin decrease shows the interference of the intrathecal administration of a potent and fast-acting $\mu$-specific opioid on Ferguson's reflex, which could directly influence labor evolution.

Two hypotheses may explain plasma oxytocin decrease in group $S$ and not in group B. The first is that Ferguson's reflex has a strong afference in pain pathways, which would justify the maintenance of oxytocin plasma concentrations in group $B$, because patients had adequate analgesia but not so deep as group S. In this case, the action of opioids and local anesthetics on oxytocin would preferably be in the spinal cord. The second hypothesis is that intrathecal sufentanil would have a rostral spread reaching the hypothalamohypophyseal axle and inhibiting oxytocin release by the posterior hypophysis lobe at the central level.

Oxytocin endogenous secretion mechanisms are highly complex and were not yet fully explained. Opioid action on hypothalamohypophyseal axle (HHA) inhibiting oxytocin release was described in experimental studies in vitro ${ }^{31}$ and in vivo in rats ${ }^{32}$.

The presence and action of $\mu$ and $\kappa$ opioids in the hypothalamohypophyseal axle has been widely shown, especially in Supra-Optic and Paraventricular Nuclei ${ }^{33}$.In Supra-Optic Nuclei magnocellular neurons, the population of $\kappa$ receptors is larger than of $\mu$ receptors and its activation promotes a decrease in electric activity and neuronal hyperpolarization inhibiting neuro-hypophysis oxytocin release ${ }^{34}$. However, in rats in the end of gestation, a decrease in $\kappa$ agonists response and an increase in $\mu$ receptors activity have been observed ${ }^{13,35}$. There are evidences of $\mu$ and $\kappa$ receptors populations in the hypophysis posterior lobe, because intra-cerebral naloxone ( $\mu$ selective antagonist) promotes oxytocin release ${ }^{31}$.

Uterine activity and oxytocin plasma levels in rats during labor decreased after subcutaneous or intra-cerebral morphine, leading to a prolonged labor and showing the inhibiting action of $\mu$ agonist opioids on central Ferguson's reflex ${ }^{36}$. Although an extensive literature on the modulation of opioids in oxytocin release in animals, few studies were carried out with humans, probably due to methodological and ethical difficulties in performing such experiments. With the development and major progresses in intrathecal analgesia with liposoluble opioids in the late 80 's, such subject became very important.

Intravenous $5 \mathrm{mg}$ morphine, administered during the first labor phase, decreases plasma oxytocin in the peripheral circulation, probably by inhibiting hypothalamohypophyseal axle release ${ }^{12}$, establishing the first correlation between clinical and experimental trials. During the second labor phase, intravenous morphine was unable to block oxytocin release, probably due to the higher stimulus intensity and the lower analgesic efficacy of intravenous morphine during this phase ${ }^{37}$. Inhibitory response to labor-induced oxytocin release, both of $\kappa$ and $\mu$ agonists, depends on the number and intensity of sensory afferences ${ }^{38}$, which confirms the possibility of oxytocin release inhibition by opioids being labor phase-dependent.

The possibility of cephalic sufentanil spread has been previously discussed. We were unable to show that sufentanil had reached the supraspinal region, but the high incidence of somnolence and pruritus and the decrease in respiratory rate in two group $S$ patients suggest the possibility of rostral spread. The fact of analgesia not changing plasma oxytocin in group B suggests that other mechanisms, other than pain blockade, are involved in oxytocin decrease in group $S$ and that sufentanil action site, for decreasing oxytocin levels, should be supraspinal.

Previous studies on the effect of epidural anesthesia with local anesthetics on oxytocin plasma levels had different results and conclusions. Patients receiving epidural analgesia had a decrease in oxytocin plasma levels after blockade ${ }^{10}$, which is different from our results. In that study, however, analgesia was induced at the end of the second labor phase, when sensory afference and the consequent oxytocin release are intense and total block with local anesthetics could show a better effect in oxytocin reflex release, which is different from our method. Although without group B oxytocin decrease in our sample, there was also no increase, as it should be expected during natural labor evolution.

Labor analgesia with intrathecal opioids does not produce loss of abdominal press and does not relax pelvis muscles. Such factors could cause rotation dystocia and prolong labor, which could explain a faster labor evolution with such technique. In spite of the seemingly labor maintenance, the effects of oxytocin levels decrease in uterine contractility and labor evolution were not the objective of this study. Uterine contractility control mechanisms are complex and partially explained. Other factors, such as decreased catecholamine plasma concentrations and number of oxytocin receptors in the myometrium may override oxytocin decrease.

Concluding, intrathecal sufentanil decreases oxytocin plasma concentrations. The possible action mechanism is the release inhibition of hypothalamohypophysial axle. The confirmation of the action mechanism and of the influence on uterine contractions needs additional studies with a specific method. 


\section{REFERÊNCIAS - REFERENCES}

01. Costa A, De Filippis V, Voglino M et al - Adrenocorticotropic hormone and catecholamines in maternal, umbilical and neonatal plasma in relation to vaginal delivery. J Endocrinol Invest, 1988;11:703-709.

02. Carr BR, Parker CRJ, Madden JD et al - Maternal plasma adrenocorticotropin and cortisol relationship throughout human pregnancy. Am J Obstet Gynecol, 1981;139:416-422.

03. McLean M, Thompson D, Zhang $\mathrm{H}$ et al - Corticotrophin-releasing hormone and $\beta$-endorphin in labour. Eur $\mathrm{J}$ Endocrinol, 1994;131:167-172.

04. Myers RE - Maternal psychological stress and fetal asphyxia: a study in the monkey. Am J Obstet Gynecol, 1975;122:47-59.

05. Ramin SM, Porter JC, Gilstrap LC et al - Stress hormones and acid-base status of human fetuses at delivery. J Clin Endocrinol Metab, 1991;73:182-186.

06. Chen DC, Nommsen -Rivers L, Dewey KG et al - Stress during labor and delivery and early lactation performance. Am J Clin Nutr, 1998;68:335-344.

07. Mc Neilly AS, Robinson IC, Houston MJ et al - Release of oxytocin and prolactin in response to suckling. Br Med J, 1983;286: 257-259.

08. Wotjak CT, Ganster J, Kohl G et al - Dissociated central and peripheral release of vasopressin, but not oxytocin, in response to repeated swin stress: new insights into the secretory capacities of peptidergic neurons. Neuroscience, 1998;85:1209-1222.

09. Scull TJ, Hemmings GT, Carli F et al - Epidural analgesia in early labour blocks the stress response but uterine contrations remain unchanged. Can J Anaesth, 1998;45:626-630.

10. Goodfellow CF, Hull MG, Swaab DF et al - Oxytocin deficiency at delivery with epidural analgesia. Br J Obstet Gynaecol, 1983;90:214-219.

11. Behrens O, Goeschen K, Luck HJ et al - Effects of lumbar epidural analgesia on prostaglandin F2alpha release and oxytocin secretion during labor. Prostaglandins, 1993;45:285-296.

12. Lindow SW, van der Spuy ZM, Hendricks MS et al - The effect of morphine and naloxone administration on plasma oxytocin concentratios in the first stage of labour. Clin Endocrinol, 1992;37: 349-353.

13. Russell JA, Leng G, Bicknell RJ - Opioid tolerance and dependence in the magnocellular oxytocin system: a physyological mechanism? Exp Physiol, 1995;80:307-340.

14. Vieira JGH - Radioimunoensaio do cortisol sérico, Tese de Mestrado, São Paulo, Escola Paulista de Medicina, 1977;1-67.

15. Shnider SM, Wright RG - Hypotension and Regional Anesthesia in Obstetrics, em: Shnider SM, Levingson G - Anesthesia for Obstetrics. $3^{\text {rd }}$ Ed, Baltimore - USA, Willians and Wilkins, 1993; 397-406.

16. Norris M C, Grieco W M, Borkowski M et al - Complications of labor analgesia: epidural versus combined spinal epidural techniques. Anesth Analg, 1994;79:529-537.

17. D'Angelo R, Anderson MT, Philip J et al - Intratecal sufentanil compared to epidural bupivacaine for labor analgesia. Anesthesiology, 1994;80:1209-1215

18. Souza H, Stiller R - Cisternal CSF and arterial plasma levels of fentanyl, alfentanil and sufentanil after lumbar epidural injection. Anesthesiology, 1989;71:A838.

19. Ferouz F, Norris MC, Arkoosh VA et al - Baricity, needle direction, and intrathecal sufentanil labor analgesia. Anesthesiology, 1997;86:592-598.
20. Richardson MG, Wissler R - Densities of dextrose-free intrathecal local anesthetics, opioids, and combinations measured at $37^{\circ} \mathrm{C}$. Anesth Analg, 1997;84:95-99.

21. Chaim W, Mazor M - The relationship between hormones and human parturiation. Arch Gynecol Obstet, 1998;262:43-51.

22. Wu W, Nathanielsz PW - Changes in oxytocin receptor messenger RNA in the endometrium, myometrium, mesometrium, and cervix of sheep in late gestation and during spontaneous and cortisol-induced labor. J Soc Gynecol Investig, 1994;1:191-196.

23. Wood CE, Keller-Wood M - Induction of parturiation by cortisol: effects on negative feedback sensitivity and plasma CRF. J Dev Physiol, 1991;16:287-292.

24. Patel FA, Clifton VL, Chwalisz K et al - Steroid regulation of prostaglandin dehydrogenase activity and expression in human term placenta and chorio-decidua in relation to labor. J Clin Endocrinol Metab, 1999;84:291-299.

25. Reynolds JL - Post-traumatic stress disorder after childbirth: the phenomenon of traumatic birth. CMAJ, 1997;156:831-835.

26. Mahomed K, Gulmezoglu AM, Nikodem VC et al - Labor experience, maternal mood and cortisol and catecholamine levels in low-risk primiparous women. J Psychosom Obstet Gynaecol, 1995;16:181-186.

27. Bacigalupo G, Riese S, Rosendahl H et al - Quantitative relationships between pain intensities during labor and beta-endorphin and cortisol concentration in plasma. J Perinat Med, 1990;18:289-296.

28. Cover PO, Buckingham JC - Effects of seletive opioid-receptor blockade on the hypothalamo-pituitary-adrenocortical responses to surgical trauma in the rat. J Endocrinol, 1988;121: 213-220.

29. Chard T, Gibbens GL - Spurt release of oxytocin during surgical indution of labor in women. Am J Obstet Gynecol, 1983;147: 678-680.

30. Gazarek F, Pohanka J, Talãs M et al - Plasma oxytocin and oxytocinase levels in thrird trimester of pregnancy and at labour. Endocrinol Exp, 1976;10:283-287.

31. Bicknell RJ, Chalman C, Leng G - Effects of opioid agonist and antagonist on oxytocin and vasopressin release in vitro. Neuroendocrinology, 1985;41:142-148.

32. Haldar J, Bade V - Involvement of opioid peptides in the inhibition of oxytocin release by heat stress in lactating mice. Proc Soc Exp Biol Med, 1981;168:10-14.

33. Rossier J, Battenberg E, Pittman Q et al - Hypothalamic enkephalin neurones may regulate the neurohypophysis. Nature, 1979;277:653-655.

34. Sumner BEH, Coombes JE, Pumford KM et al - Opioid receptor subtypes in the supraoptic nucleus and posterior pituitary gland of morphine-tolerant rats. Neuroscience, 1990;37:635-645.

35. Douglas AJ, Neumann I, Meeren HK et al - Central endogenous opioid inhibition of supraoptic oxytocin neurons in pregnant rats. J Neurosci, 1995;15:5049-5057.

36. Russel JA, Gosden RG, Humphreys EM et al - Interruption of parturiation in rats by morphine: a result of inhibition of oxytocin secretion. J Endocrinol, 1988;121:521-536.

37. Lindow SW, Van Der Spuy ZM, Hendricks MS et al - The effect of morphine and naloxone administration on the maternal oxytocin concentration in late pregnancy. Clin Endocrinol, 1993;39: 671-675.

38. Pumford KM, Leng G, Russell JA - Excitatory sinaptic drive offsets opiod inhibition of oxytocin neurons. Ann N Y Acad Sci, 1993;689:656-658. 


\section{RESUMEN}

Stocche RM, Garcia LV, Klamt JG - Efectos de la Analgesia del Sufentanil por Vía Subaracnóidea y Bupivacaína a 0,25\% por Vía Peridural sobre las Concentraciones Plasmáticas de Ocitocina y Cortisol en Embarazadas en Trabajo de Parto

Justificativa y Objetivos - El sufentanil por vía subaracnóidea promueve analgesia del trabajo de parto comparable a la peridural con anestésicos locales. Las dos técnicas diminuyen algunos parámetros de la respuesta neuroendócrina al dolor del parto, y ambas pueden atenuar la liberación refleja de ocitocina en animales. El objetivo de este estudio fue comparar los efectos de las dos técnicas en las concentraciones plasmáticas de ocitocina (CPO) y cortisol (CPC) durante el trabajo de parto.

Método - Treinta parturientas con 4-7 cm de dilatación, fueron escogidas en este estudio de forma aleatoria y encubierta. En el grupo S recibieron sufentanil $(10 \mu \mathrm{g})$, por via subaracnoidea $y$ en el grupo $B$ recibieron bupivacaína a 0,25\% por vía peridural. La analgesia fue medida usando Escala Analógica Visual y muestras sanguíneas de ocitocina y cortisol fueron obtenidas antes, 30 y 60 minutos después la administración de las drogas. As CPO y CPC fueron determinadas por medio de la técnica de radioinmunoensayo.

Resultados - La analgesia en el grupo S fue más intensa con 30 minutos. Las CPO y CPC en los dos grupos fueron semejantes antes de la analgesia. En el grupo $S$, hubo diminución significativa de la CPO a los 60 minutos y CPC a los 30 y 60 minutos, en cuanto en el grupo $B$, CPO y CPC permanecieron estables durante todo el estudio.

Conclusiones - En las condiciones de este estudio, la analgesia peridural con bupivacaína estuvo asociada a la manutención de las concentraciones plasmáticas de cortisol y ocitocina. Contrariamente, la analgesia promovida por el sufentanil, por vía subaracnóidea, fue más intensa y produjo diminución en las concentraciones de estas hormonas. 